\title{
Non-extensivity and long-range correlations in the earthquake activity at the West Corinth rift (Greece)
}

\author{
G. Michas ${ }^{1}$, F. Vallianatos ${ }^{1,2}$, and P. Sammonds ${ }^{1}$ \\ ${ }^{1}$ Institute for Risk and Disaster Reduction, University College London, Gower Street, London, WC1E 6BT, UK \\ ${ }^{2}$ Laboratory of Geophysics and Seismology, Technological Educational Institute of Crete, Chania, 73133, Crete, Greece
}

Correspondence to: G. Michas (georgios.michas.10@ucl.ac.uk)

Received: 16 February 2013 - Revised: 1 June 2013 - Accepted: 2 August 2013 - Published: 27 September 2013

\begin{abstract}
In the present work the statistical properties of the earthquake activity in a highly seismic region, the West Corinth rift (Central Greece), are being studied by means of generalized statistical physics. By using a dataset that covers the period 2001-2008, we investigate the earthquake energy distribution and the distribution of the time intervals (interevent times) between the successive events. As has been reported previously, these distributions exhibit complex statistical properties and fractality. By using detrended fluctuation analysis (DFA), a well-established method for detection of long-range correlations in non-stationary signals, it is shown that long-range correlations are also present in the earthquake activity. The existence of these properties motivates us to use non-extensive statistical physics (NESP) to investigate the statistical properties of the frequencymagnitude and the interevent time distributions, along with other well-known relations in seismology, such as the gamma distribution for interevent times. The results of the analysis indicate that the statistical properties of the earthquake activity can be successfully reproduced by means of NESP and that the earthquake activity at the West Corinth rift is correlated at all-time scales.
\end{abstract}

\section{Introduction}

Earthquakes exhibit complex phenomenology that is related to the deformation and sudden rupture of parts of the earth's brittle crust due to external forces arising from plate tectonic motions. The lithosphere can be regarded as a large nonlinear dynamical system (Keilis-Borok, 1990) that interacts on a wide range of space and timescales (Main, 1996; Turcotte, 1997). Complex correlations in the earthquake activity have been recognized and reported in a number of studies (Main, 1996; Turcotte, 1997; Bak et al., 2002; Rundle et al., 2003; Varotsos et al., 2005; Lennartz et al., 2011). Despite this complexity, there are some universal scaling relations that characterize earthquakes. The best known is the GutenbergRichter scaling relation (Gutenberg and Richter, 1944) that indicates a fractal power-law earthquake size distribution. Scale-invariance and fractality have been also reported for the fracture/fault populations and the spatiotemporal properties of the earthquake activity (e.g. Main, 1996; Turcotte, 1997), leading to the consideration of a self-organized critical (SOC) mechanism in the origin of seismogenesis (e.g. Bak and Tang, 1989; Main, 1996; Telesca et al., 2001; Sornette, 2004).

Since the underlying physics that control the nucleation and evolution of the earthquake activity still remains elusive, statistical physics may be used to derive the macroscopic laws from the specification of the relevant microscopic elements and their interactions. In this direction, statistical physics aims to explain the complex patterns that are evident from the fault and earthquake populations in a variety of scales (Main, 1996; Rundle et al., 2003).

In this work, considering the fractal properties of earthquakes, we use generalized statistical physics to study the statistical properties of the earthquake activity in a high seismic risk zone, the West Corinth rift (central Greece). This approach refers to non-extensive statistical physics (NESP) that was proposed by Tsallis (1988) as a generalization of the classic Boltzmann-Gibbs statistical physics. NESP has been inspired by fractals and is a consistent theoretical framework for the analysis of nonlinear dynamical systems that exhibit fractal structures and long-range correlations among their elements. The NESP concept has been 
recently applied to various fields of geophysics including earthquakes (Abe and Suzuki, 2003; 2005; Telesca, 2010a, b, 2011), fault lengths distribution (Vallianatos and Sammonds, 2011), plate tectonics and geodynamics (Vallianatos and Sammonds, 2010, 2013), rock physics (Vallianatos et al., 2011, 2012a; Vallianatos and Triantis, 2012), natural hazards (Vallianatos, 2009, 2013; Chen et al., 2011) and geomagnetic reversals (Vallianatos, 2011).

The aim of the present work is to study the statistical properties of the earthquake activity at the West Corinth rift (central Greece) by means of NESP principles and to establish a firm basis for the statistical physics interpretation of the earthquake phenomenon. We first use detrended fluctuation analysis (DFA) to investigate the type of correlations that might be present in the evolution of the earthquake activity at the West Corinth rift. DFA is a reliable statistical method for the detection of long-range correlation in non-stationary fluctuating signals and has been widely applied to diverse fields including geophysics (Varotsos et al., 2002; Telesca et al., 2003; Lennartz et al., 2008; Telesca and Lovallo, 2009). We then investigate the frequency-magnitude and the interevent time distributions in a NESP framework for the period 20012008 and then compare the results with other well-known distributions in seismology, such as the $\mathrm{G}-\mathrm{R}$ relation for the earthquake energy distribution (Gutenberg and Richter, 1944) and the gamma distribution for the probability density of the time intervals between the successive earthquakes (Corral, 2004). The results indicate that the earthquake activity is correlated at all-time scales and that its statistical properties can be successfully reproduced using NESP.

\section{Dataset}

The Corinth rift constitutes one of the most seismotectonically active areas in Europe (Makropoulos et al., 1989; Ambraseys and Jackson, 1990) due to a significant continental $\mathrm{N}-\mathrm{S}$ extension of about 13 and $6 \mathrm{~mm} \mathrm{yr}^{-1}$ at the west and east part, respectively (Clarke et al., 1997). This extension is expressed by important normal faults on the north and south margin of the basin of an E-W general direction, creating an asymmetric tectonic graben with the southern footwall being uplifted (Armijo et al., 1996). The seismicity of the area includes 5 main earthquakes of magnitude greater than 5.8 since 1960. The last major earthquake was the $1995 \mathrm{Ai}-$ gion earthquake $\left(M_{\mathrm{S}}=6.2\right.$, Bernard et al., 1997) and since then the seismic activity has been dominated by small- to medium-sized earthquakes.

Here, we consider the crustal (depth $\leq 35 \mathrm{~km}$ ) earthquake activity that occurred during the 2001-2008 period at the West Corinth rift. The activity is concentrated in its great majority in a narrow $52.23 \mathrm{~km} \times 44.4 \mathrm{~km}$ zone (area between $38.1-38.5^{\circ} \mathrm{N}$ and $21.8-22.4^{\circ} \mathrm{E}$ ) (Fig. 1). A catalogue compiled by the CRL network (http://crlab.eu/), a dense network located at the west part of the Corinth rift, is used. During this period, sudden seismic crises of swarm-like character

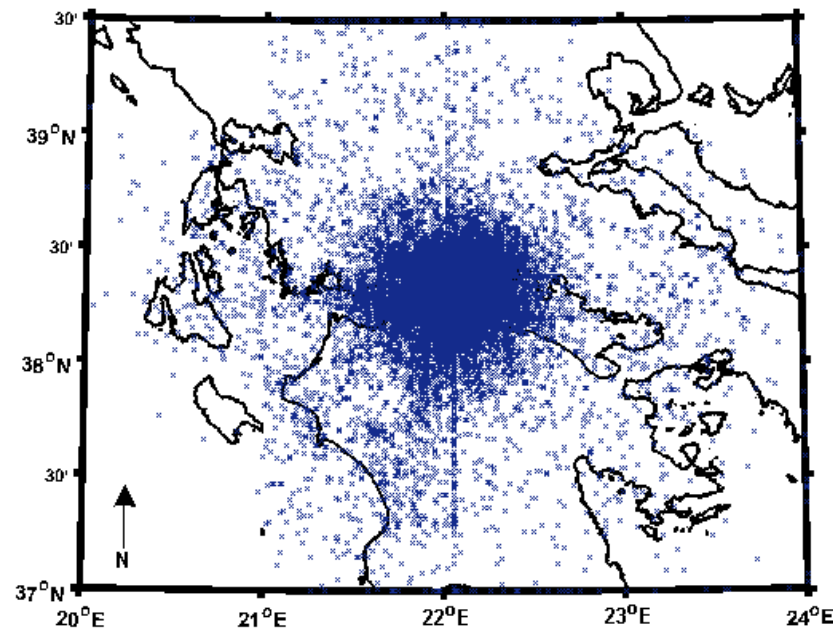

Fig. 1. Map of central Greece with the earthquakes recorded from the CRL network during 2001-2008.

are frequent (Fig. 2a) (Bourouis and Cornet, 2009; Pacchiani and Lyon-Caen, 2010), with earthquake magnitudes not exceeding the value of $4\left(M_{\mathrm{L}}\right)$ (Fig. $\left.2 \mathrm{~b}\right)$.

We have applied the Gutenberg-Richter (G-R) empirical relationship (Gutenberg and Richter, 1944) in order to detect the lower magnitude for which the dataset can be considered complete $\left(M_{\mathrm{c}}\right)$ (Fig. 2c). The G-R relation expresses a power-law dependence of the cumulative number of earthquakes $N$ greater than a threshold magnitude $M$

$N(>M) \sim 10^{-b M}$,

where $b$ is the slope of the frequency-magnitude distribution and describes the size distribution of the earthquake events. The application of the $\mathrm{G}-\mathrm{R}$ relation to the dataset resulted in a $b$ value of $b=1.51 \pm 0.03$ and a lower magnitude of $M_{\mathrm{c}}=1.4$ for which the G-R relation holds. The relatively high $b$ value that is found here reflects the domination of small to moderate magnitude earthquakes and the absence of larger magnitude earthquakes for the period considered here, in comparison to the $b$ value of $b=1.08 \pm 0.05$ that has been found by Telesca et al. (2002) for the 1983-2000 period. A closer inspection of Fig. $2 \mathrm{~b}$ reveals a significant increase at the lower magnitude earthquake events after 2001, implying that different $M_{\mathrm{c}}$ may exist at the dataset for various time periods. The application of the G-R relation to different time windows reveals that for 2001 the magnitude of completeness is $M_{\mathrm{c}}=1.6$ and for 2002-2008 $M_{\mathrm{c}}=1.2$. Considering the earthquakes of magnitude $M \geq M_{\mathrm{c}}$, a dataset of 37622 earthquake events emerges for the period 2001-2008.

\section{Detrended fluctuation analysis}

Detrended fluctuation analysis (DFA), originally introduced by Peng et al. (1994), is a scaling technique based on random 


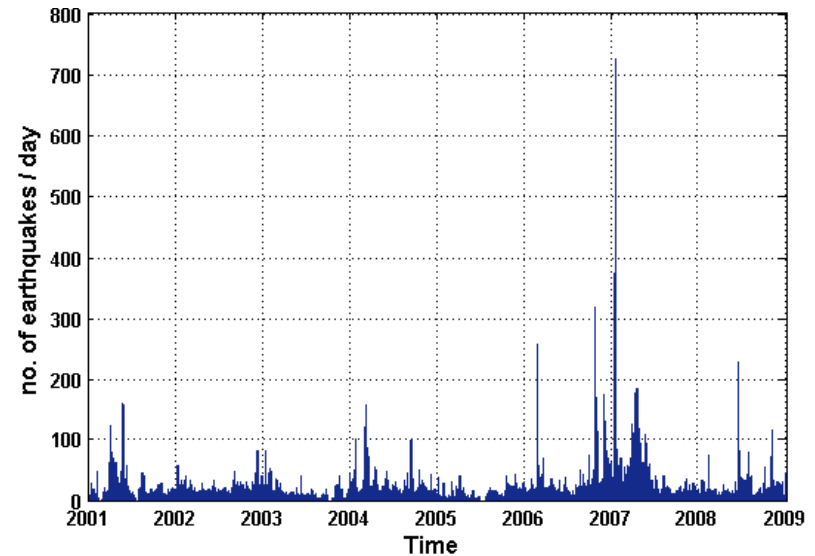

(a)

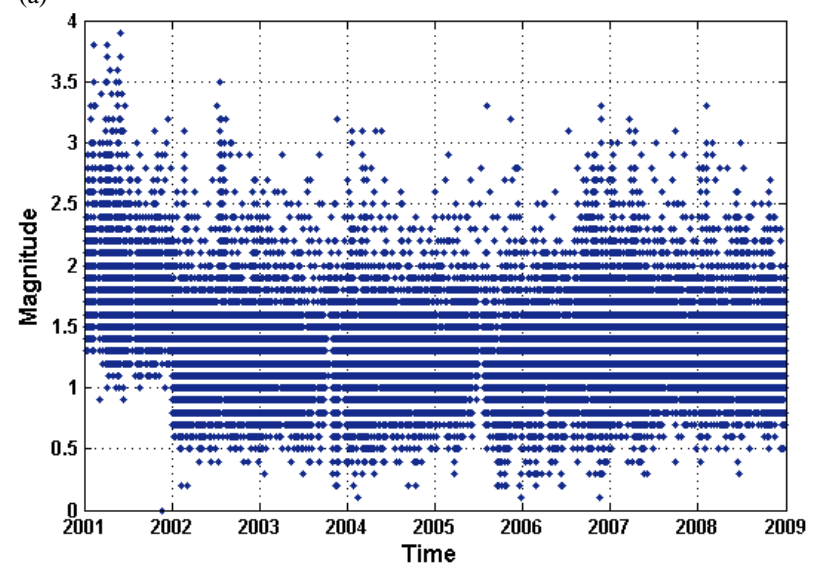

(b)

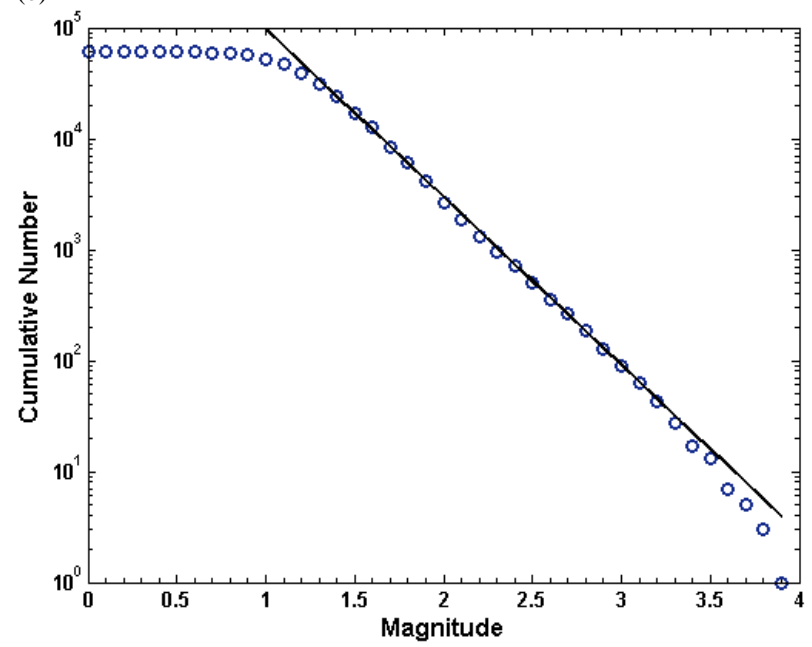

(c)

Fig. 2. (a) Seismicity rate per day, (b) magnitude $\left(M_{\mathrm{L}}\right)$ range per day, and (c) frequency-magnitude distribution for the period 20012008 at the West Corinth rift. The solid line represents the G-R relation.

walk theory that is used for the estimation of long-range correlations in non-stationary fluctuating signals. In recent years, DFA has been widely applied to various dynamical systems where long-range correlations are of interest (e.g.
Bashan et al., 2008). The advantage of DFA over other scaling techniques is that it can reliably estimate and quantify the correlations in non-stationary fluctuating signals embedded in polynomial trends, avoiding long-range correlations that are artifacts of non-stationarity (Hu et al., 2001; Chen et al., 2002).

For the application of DFA, a fluctuating signal $u(i)$ of total length $N(i=1, \ldots, N)$ is first shifted by the average $<u>$ and integrated,

$y(k)=\sum_{i=1}^{k}[u(i)-\langle u\rangle]$,

with $k=1,2, \ldots, N$. Then the integrated signal $y(k)$ is divided into non-overlapping windows of equal size $n$ resulting in $N_{n}=(N / n)$ segments. Since the length $N$ of the signal is not always a multiple of the window size $n$, a short part at the end of the integrated signal $y(k)$ is not included in the analysis. In order not to disregard this part, the procedure is repeated starting from the end of $y(k)$, resulting in this way into $v=2 N_{n}$ total segments (Kantelhardt et al., 2001). In each of the $2 N_{n}$ segments, the integrated signal $y(v)$ is fitted to a polynomial function $y_{n}(v)$ that represents the local trend. For different orders $l$ of the polynomial function $y_{n}(v)$, different orders of DFA are obtained and the method is denoted as DFA- $l$. Then the integrated signal $y(v)$ is detrended by subtracting the local trend $y_{n}(v)$ in each window and the root-mean-square fluctuation $F(n)$ is calculated,

$F(n)=\sqrt{\frac{1}{2 N_{n}} \sum_{\nu=1}^{2 N_{n}}\left[y(v)-y_{n}(v)\right]^{2}}$.

These computations are repeated for various window sizes $n$ to provide a relationship between $F(n)$ and $n$. If a powerlaw relation between $F(n)$ and $n$ exists, it indicates the presence of scaling: $F(n) \sim n^{\alpha}$. The parameter $a$ is the correlation exponent that represents the slope of the line that fits $\log F(n)$ to $\log n$. For uncorrelated signals, $a=0.5$ while for $a>0.5$ the signal is correlated and for $a<0.5$ anticorrelated. The $a=1$ case indicates flicker-noise dynamics and $a=1.5$ is characteristic for Brownian-like dynamics (Peng et al., 1995). Artificial crossovers in the scaling behavior of $F(n)$ can be created due to trends in the fluctuating signal and the DFA- $l$ method should be applied for different orders $l$ of the detrending polynomial function in order to gain information on the order of the trends and to obtain a reliable estimate of the correlations in the signal (Kantelhardt et al., 2001; Xu et al., 2005).

We have applied DFA on the interevent time series $\tau_{i}$ in order to identify the type of correlations that are present in this highly fluctuating series that characterize the temporal evolution of the earthquake activity at the West Corinth rift. The non-stationary and highly fluctuating behavior of the interevent time series can be visualized in Fig. 3, where the 
time of the next earthquake greatly varies from the previous one.

In Fig. 4 the logarithm of the root-mean-square fluctuation $F(n)$ that resulted for various window sizes $n$ (up to $N / 10$ ) after the application of DFA- $l(l=1,2,3)$ to the interevent time series for $M \geq M_{\mathrm{c}}$, is presented. The value of the correlation exponent $a$ is $>0.5$ indicating that the interevent time series is correlated. A change in the scaling behavior of $F(n)$ also occurs for all the different orders of DFA, resulting in greater values of the correlation exponent $\alpha$ for greater window sizes $n$. We have also applied DFA- $l(l=1,2,3)$ to a randomly shuffled copy of the original interevent time series (Peng et al., 1995). In this way any correlations due to the order of the original series are destroyed and the surrogate series should exhibit a correlation exponent $a=0.5$. The resulted $F(n)$ in this case exhibits a correlation exponent $a=0.5$ (Fig. 4), confirming that the scaling behavior of $F(n)$ for the original interevent time series is due to correlations in the time of the successive earthquakes. This result indicates a persistent behavior, where short interevent times are more likely to be followed by short ones and large interevent times by large ones.

\section{Non-extensive statistical physics concept}

A monument of contemporary physics is Boltzmann-Gibbs (BG) statistical physics. This refers to the Boltzmann-Gibbs entropy $S_{\mathrm{BG}}$ that in the discrete form reads as

$S_{\mathrm{BG}}=-k_{B} \sum_{i=1}^{W} p_{i} \ln p_{i}$,

where $k_{B}$ is Boltzmann's constant, $p_{i}$ is a set of probabilities and $W$ is the total number of microscopic configurations. One of the main characteristics of $S_{\mathrm{BG}}$ is additivity, namely the proportionality to the number of the systems' elements. In 1988 Tsallis (Tsallis, 1988) proposed a generalized form of $S_{\mathrm{BG}}$, the nonadditive Tsallis entropy $S_{q}$, which reads as

$$
S_{q}=k_{B} \frac{1-\sum_{i=1}^{W} p_{i}^{q}}{q-1}, q \in R .
$$

This particular entropic form depends on the value of the entropic index $q$, which is a measure of the non-extensivity of the system. For the particular case of $q=1, S_{1}=S_{\mathrm{BG}}$ and Eq. (4) is obtained. Although Tsallis entropy shares a lot of common properties with Boltzmann-Gibbs entropy, $S_{\mathrm{BG}}$ is additive, whereas $S_{q}(q \neq 1)$ is nonadditive (Tsallis, 2009). According to this property, $S_{\mathrm{BG}}$ exhibits only short-range correlations and the total entropy depends on the size of the systems' elements. On the other hand, $S_{q}$ allows all-length scale correlations and seems more adequate for complex dynamical systems when long-range correlations between the elements of the system are present.

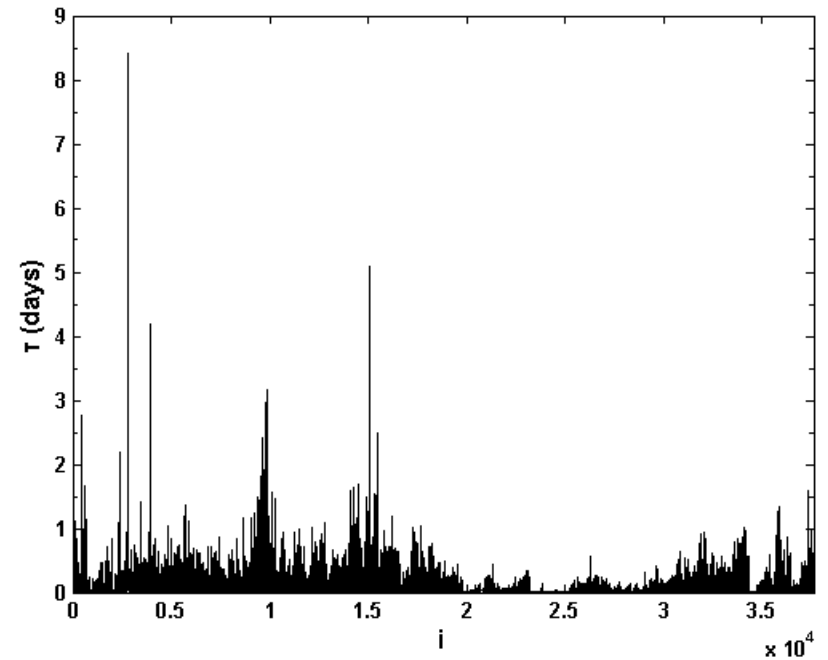

Fig. 3. The interevent time series $\tau$ of the earthquake activity for $M \geq M_{\mathrm{c}}$ versus their index number $i(i=1, \ldots, N$ where $N$ is the total length).

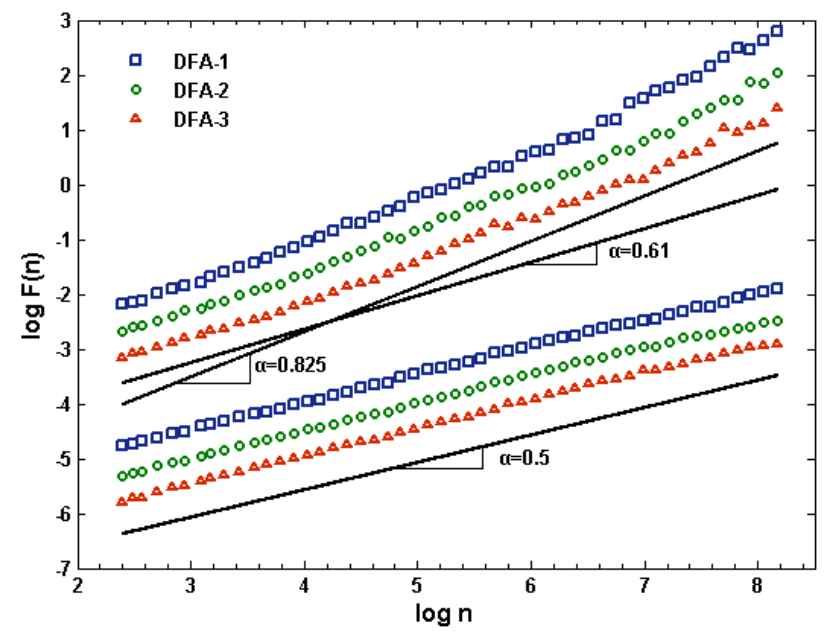

Fig. 4. The DFA- $l$ for the original inter-event time series (top) and the surrogate series (bottom). $F(n)$ has been shifted vertically for clarity. The different symbols correspond to different orders $l$ ( $l=$ $1,2,3)$ of DFA.

For any two probabilistically independent systems A and $\mathrm{B}$, i.e. if the joint probability satisfies $p_{i j}^{\mathrm{A}+\mathrm{B}}=p_{i}^{\mathrm{A}} p_{j}^{\mathrm{B}}(\forall(i j))$, Tsallis entropy $S_{q}$ satisfies

$\frac{S_{q}(\mathrm{~A}+\mathrm{B})}{k_{\mathrm{B}}}=\frac{S_{q}(\mathrm{~A})}{k_{\mathrm{B}}}+\frac{S_{q}(\mathrm{~B})}{k_{\mathrm{B}}}+(1-q) \frac{S_{q}(\mathrm{~A})}{k_{\mathrm{B}}} \frac{S_{q}(\mathrm{~B})}{k_{\mathrm{B}}}$.

The origin of nonadditivity comes from the last term on the right-hand side of this equation and is the fundamental principle of non-extensive statistical physics (Tsallis, 2009). The cases $q>1, q=1$ and $q<1$ correspond to subadditivity, additivity and superadditivity, respectively. 


\section{Earthquake energy distribution}

Earthquakes generally originate from the deformation and the sudden rupture of parts of the earth's brittle crust due to the relative motion of the tectonic plates releasing energy. The energy distribution of earthquakes exhibits power-law decay, as it is stated in the G-R relation:

$P(E) \sim E^{-\gamma}$.

This expression can be alternatively stated as Eq. (1) for the cumulative distribution of the earthquake magnitudes. Considering that the earthquake energy $E$ is related to the magnitude $M$ as $E \sim 10^{1.5 M}$ (Kanamori, 1978), Eq. (7) turns to Eq. (1) for $\gamma=1+b / 1.5$.

The mechanical phenomenology of this phenomenon is captured in simple models (e.g. Burridge and Knopoff, 1967) where a stick-slip mechanism and the friction between the faults planes have a dominant role. Consistent with this idea, Sotolongo-Costa and Posadas (2004), based on a non-extensive formalism, have developed a general physical model for earthquake dynamics that contains the G-R relation as a particular case. In this model the mechanism of earthquake triggering is based not only on the slippage of fault planes and the relative displacement due to the breakage of the asperities (e.g. De Rubeis et al., 1996), but is also caused by the fragments filling the space between fault planes. As the breakage and the displacement of the asperities and fragments cause the earthquake energy release, this energy is thought to be proportional to the volume of the fragments (Sotolongo-Costa and Posadas, 2004). Then the energy distribution function of earthquakes can be expressed in the terms of the size distribution of the fragments between the fault planes.

Sotolongo-Costa and Posadas (2004) proposed that since fragments are the result of the violent fractioning between the fault planes, then long-range interactions are expected among all the existent fragments. Fractioning is then a paradigm of non-extensivity and NESP seems more appropriate to describe the phenomenon than BG statistical physics. This is also supported by the scale-invariant properties of fractures (Krajcinovic and Van Mier, 2000) and laboratory experiments in fractured materials (Vallianatos et al., 2011, 2012a).

The maximum Tsallis entropy $S_{q}$ for the probability $p(\sigma)$ of finding a fragment of surface $\sigma$ is written as

$S_{q}=k_{B} \frac{1-\int p^{q}(\sigma) d \sigma}{q-1}$.

The sum of all the possible states in the definition of entropy is here expressed through the integration in all the sizes of the fragments. In what follows, $k_{B}$ is set as $k_{B}=1$ for the sake of simplicity. The probability $p(\sigma)$ is obtained after maximization of $S_{q}$ under the appropriate two constraints. The first is the normalization of $p(\sigma)$ :

$$
\int_{0}^{\infty} p(\sigma) d \sigma=1
$$

The second is the condition about the $q$-expectation value (Tsallis, 2009):

$$
\sigma_{q}=\langle\sigma\rangle_{q}=\frac{\int_{0}^{\infty} \sigma p^{q}(\sigma) d \sigma}{\int_{0}^{\infty} p^{q}(\sigma) d \sigma} .
$$

This last condition reduces to the definition of the mean value in the limit $q \rightarrow 1$.

By using the Lagrange multipliers technique, the functional entropy to be maximized is (Silva et al., 2006)

$\delta S_{q}^{*}=\delta\left(S_{q}+\alpha \int_{0}^{\infty} p(\sigma) d \sigma-\beta \sigma_{q}\right)=0$,

where $\alpha$ and $\beta$ are the Lagrange multipliers. After some algebra, the following expression for the fragment size distribution function can be derived (Silva et al., 2006):

$p(\sigma)=\left[1-\frac{(1-q)}{(2-q)}\left(\sigma-\sigma_{q}\right)\right]^{1 /(1-q)}$.

The proportionality between the released relative energy $E$ and the size of the fragments $r$ is now introduced as $E \sim r^{3}$ (Silva et al., 2006), in accordance to the standard definition of seismic moment scaling with rupture length (Lay and Wallace, 1995). The proportionality between the released relative energy $E$ and the three-dimensional size of the fragments $r^{3}$ now becomes

$\sigma-\sigma_{q}=\left(\frac{E}{\alpha_{E}}\right)^{2 / 3}$.

In the last equation, $\sigma$ scales with $r^{2}$ and $\alpha_{E}$ is the proportionality constant between $E$ and $r^{3}$ that has the dimension of volumetric energy density. By using the latter equation, the energy distribution function (EDF) of the earthquakes can be written on the base of the relationship between density functions of correlated stochastic variables (Telesca, 2012):

$$
\begin{aligned}
p(E) & =\frac{1}{\frac{\mathrm{d} E}{\mathrm{~d} \sigma}} p\left[\left(\frac{E}{\alpha_{E}}\right)^{2 / 3}+\sigma_{q}\right] \\
& =\frac{\mathrm{d} \sigma}{\mathrm{d} E}\left[1-\frac{(1-q)}{(2-q)}\left(\frac{E}{\alpha_{E}}\right)^{2 / 3}\right]^{\frac{1}{1-q}},
\end{aligned}
$$

where the term $\mathrm{d} \sigma / \mathrm{d} E$ can be obtained by differentiating Eq. (13):

$\frac{\mathrm{d} \sigma}{\mathrm{d} E}=\frac{2}{3} \frac{E^{-\frac{1}{3}}}{\alpha_{E}^{\frac{2}{3}}} \mathrm{~d} E$. 
The EDF now becomes (Silva et al., 2006; Telesca, 2012)

$p(E)=\frac{C_{1} E^{-\frac{1}{3}}}{\left[1+C_{2} E^{\frac{2}{3}}\right]^{1 /(q-1)}}$,

with $C_{1}=\frac{2}{3 \alpha_{E}^{\frac{2}{3}}}$ and $C_{2}=-\frac{(1-q)}{(2-q) \alpha_{E}^{\frac{2}{3}}}$

In the latter expression, the probability of the energy is $p(E)=n(E) / N$, where $n(E)$ corresponds to the number of earthquakes with energy $E$ and $N$ is the total number of earthquakes. A more viable expression can now be obtained by introducing the normalized cumulative number of earthquakes given by the integral of Eq. (16):

$$
\frac{N\left(E>E_{\mathrm{th}}\right)}{N}=\int_{E_{\mathrm{th}}}^{\infty} p(E) \mathrm{d} E,
$$

where $N\left(E>E_{\mathrm{th}}\right)$ is the number of earthquakes with energy $E$ greater than the threshold energy $E_{\text {th }}$ and $N$ the total number of earthquakes. Substituting Eq. (16) in Eq. (17) the following expression is derived:

$\frac{N\left(E>E_{\mathrm{th}}\right)}{N}=\left[1-\left(\frac{1-q_{E}}{2-q_{E}}\right)\left(\frac{E}{\alpha_{E}}\right)^{\frac{2}{3}}\right]^{\frac{2-q_{E}}{1-q_{E}}}$.

Now the latter expression can be written in terms of the earthquake magnitude $M$, if we consider that $E$ is related to $M$ as $M=\frac{2}{3} \log (E)$ (Kanamori, 1978). Then Eq. (18) becomes

$\frac{N(>M)}{N}=\left[1-\left(\frac{1-q_{E}}{2-q_{E}}\right)\left(\frac{10^{M}}{\alpha_{E}^{2 / 3}}\right)\right]^{\frac{2-q_{E}}{1-q_{E}}}$.

This relationship describes (from the first principles and in NESP formalism) the cumulative distribution of the number of earthquakes $N$ greater than the threshold magnitude $M$ in a seismic region, normalized by the total number of earthquakes. The constant $a_{E}$ expresses the proportionality between the released energy $E$ and the fragment size $r$, while $q$ is the entropic index that from now on we will refer to as $q_{E}$ for reasons of clarity. This model was recently applied to regional seismicity, covering diverse tectonic regions (Silva et al., 2006; Vilar et al., 2007; Telesca, 2010a, b, 2011) and volcano related seismicity (Telesca, 2010a; Vallianatos et al., 2013) and has been found to describe appropriately the energy distribution in a wider detectable range of magnitudes than the G-R relation. Above some threshold magnitude, the $\mathrm{G}-\mathrm{R}$ relation can be deduced as a particular case of the non-extensive model of Eq. (19) for $b=\left(2-q_{E}\right) /\left(q_{E}-1\right)$ (Telesca, 2012).

In real earthquake catalogues the threshold magnitude $M_{0}$, i.e. the minimum magnitude $M_{0}$ of the catalogue, has to be taken into account and Eq. (19) should be slightly changed to (Telesca, 2012)

$\frac{N(>M)}{N}=\left[\frac{1-\left(\frac{1-q_{E}}{2-q_{E}}\right)\left(\frac{10^{M}}{\alpha_{E}^{2 / 3}}\right)}{1-\left(\frac{1-q_{E}}{2-q_{E}}\right)\left(\frac{10^{M_{0}}}{\alpha_{E}^{2 / 3}}\right)}\right]^{\frac{2-q_{E}}{1-q_{E}}}$.

We have applied the non-extensive model of Eq. (20) to the magnitude distribution of the earthquake activity at the West Corinth rift. The model describes quite well the normalized cumulative magnitude distribution for the values of $q_{E}=1.37 \pm 0.01$ and $\alpha_{E}=19.05 \pm 6.86$ and for $M \geq M_{0}=$ 1 (Fig. 5a). In comparison to the G-R relation (Eq. 1), the model of Eq. (20) provides a better descriptor for the data in the lower magnitude range ( $M \leq 1.3$ ), while for $M \geq 1.4$ the magnitude distribution decays as a power-law (Fig. 5a). This is also evident from the energy distribution function (EDF) that is plotted in Fig. 5b. The non-extensive model, in this case according to Eq. (16) and for the same values of $q_{E}$ and $\alpha_{E}$ as previously, describes well the EDF. Above some threshold energy the EDF decays as power-law with exponent $\gamma=1.98$ (Eq. 7).

\section{Interevent time distribution}

DFA analysis indicated that long-range correlations exist at the interevent time series. This property should be also reflected in the probability distribution function, where a deviation from the ordinary exponential function and BG statistical physics, which are commonly used to describe uncorrelated processes, can be expected. Then NESP may provide a more appropriate framework for a statistical physics approach to the time of earthquake occurrences. In this direction, Abe and Suzuki (2005) have proposed that the cumulative interevent time distribution $P(>\tau)$, i.e. the probability finding an event greater than the interevent time $\tau$, follows a $q$-exponential distribution with $q>1$. This function has been frequently used in studies of complex dynamical systems (Gell-Mann and Tsallis, 2004; Tsallis, 2009), where long-range correlations lead to asymptotic power-law behavior. The $q$-exponential distribution for $q>1$ corresponds to a Zipf-Mandelbrot-type distribution (Mandelbrot, 1983) and exhibits in a novel manner the scale-free nature of seismicity (Abe and Suzuki, 2005). This approach has been successfully applied to global (Vallianatos and Sammonds, 2013), regional (Abe and Suzuki, 2005; Darooneh and Dadashinia, 2008) and laboratory scale (Vallianatos et al., 2012a), including the Aigion earthquake aftershock sequence (Vallianatos et al., 2012b) and volcano-related seismicity (Vallianatos et al., 2013).

The $q$-exponential distribution is derived after maximization of the Tsallis entropy $S_{q}$ under the appropriate constraints with the Lagrange multipliers method (Abe and Suzuki, 2005; Tsallis, 2009). In terms of the probability 


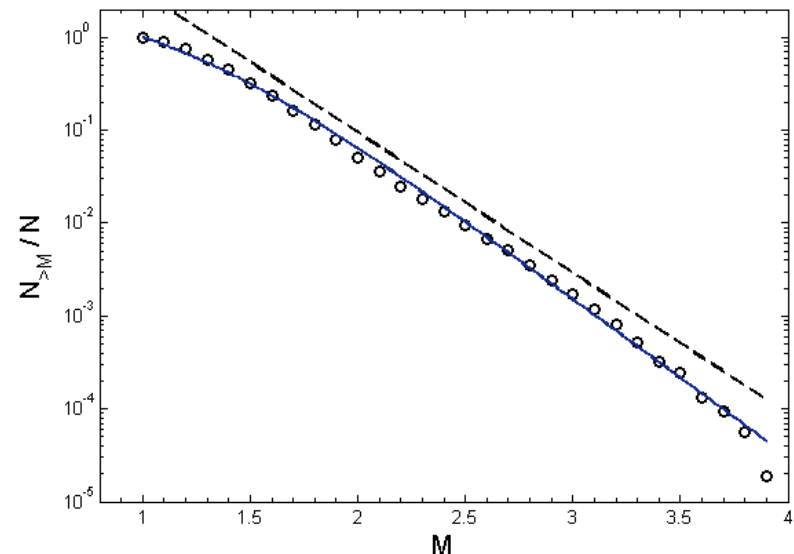

(a)

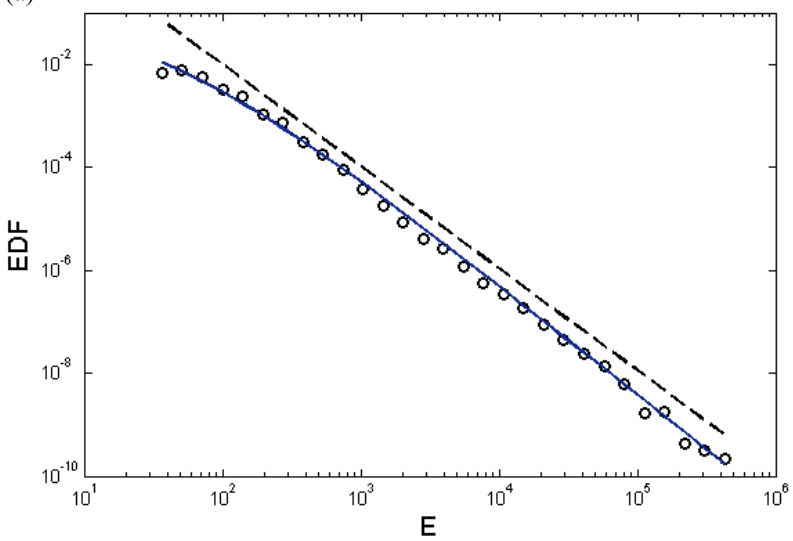

(b)

Fig. 5. (a) Normalized cumulative magnitude distribution for $M \geq$ $M_{O}=1$ (circles) and the model of Eq. (20) (solid line) for the values of $q_{E}=1.37 \pm 0.01$ and $\alpha_{E}=19.05 \pm 6.86$. The dashed line represents the $\mathrm{G}-\mathrm{R}$ relation for $b=1.51 \pm 0.03$. (b) The corresponding energy distribution function (circles). The fitted curve (solid line) is according to Eq. (16) for the same values of $q_{E}$ and $\alpha_{E}$ as previously and the G-R relation (Eq. 7) for $\gamma=1.98 \pm 0.03$.

distribution of the interevent times $p(\tau)$ and for $k_{B}=1, S_{q}$ reads as

$S_{q}=\frac{1-\int_{0}^{\infty} p^{q}(\tau) d \tau}{q-1}$

The two constraints refer to the normalization of $p(\tau)$

$\int_{0}^{\infty} p(\tau) d \tau=1$

and the condition about the $q$-expectation value (Tsallis, 2009)

$\tau_{q}=\langle\tau\rangle_{q}=\frac{\int_{0}^{\infty} \tau p^{q}(\tau) d \tau}{\int_{0}^{\infty} p^{q}(\tau) d \tau}$.
The functional that we require optimizing is in this case:

$\delta S_{q}^{*}=\delta\left(S_{q}-a \int_{0}^{\tau_{\max }} p(\tau) d \tau-\beta^{*} \tau_{q}\right)=0$,

where $\alpha$ and $\beta^{*}$ represent the Lagrange multipliers. Thus we obtain the physical probability:

$p(\tau)=\frac{\left[1-(1-q) B_{q} \tau\right]^{1 / 1-q}}{Z_{q}}=\frac{\exp \left(-B_{q} \tau\right)}{Z_{q}}$,

where the $q$-exponential function is defined as

$e_{q}(x)=[1+(1-q) x]^{1 /(1-q)}$, for $1+(1-q) x \geq 0$

and

$e_{q}(x)=0$, for $1+(1-q) x<0$,

$Z_{q}$ is the $q$ partition function

$Z_{q}=\int_{0}^{\tau_{\max }} \exp _{q}\left(-B_{q} \tau\right) d \tau$

where $B_{q}=\frac{\beta^{*}}{C_{q}-(1-q) \beta^{*} \tau_{q}}$ and $C_{q}=\int_{0}^{\tau_{\max }} p^{q}(\tau) d \tau$. The inverse of $e_{q}(x)$ is the $q$-logarithmic distribution $\ln _{q}(x)$ :

$\ln _{q}(x)=\frac{1}{1-q}\left(x^{1-q}-1\right)$

that is linear with $x$ for the appropriate $q$-value. In the limit $q \rightarrow 1$, the ordinary exponential and logarithmic functions are obtained from the $e_{q}(x)$ and $\ln _{q}(x)$ functions, respectively.

The cumulative distribution $P(>\tau)$ that is associated to the physical probability $p(\tau)$ of Eq. (25) is given by

$P(>\tau)=\int_{\tau}^{\infty} p(\tau) \mathrm{d} \tau=\exp _{q^{\prime}}\left(-B_{q^{\prime}} \tau\right)$,

$q^{\prime}=1 /(2-q)$ and $B_{q^{\prime}}=(2-q) / B_{q}$ (Picoli et al., 2009). The cumulative distribution $P(>\tau)$ also exhibits the $q$ exponential form as the physical probability $p(\tau)$.

The form of $P(>\tau)$ given in Eq. (29) is equivalent to the form derived by Abe and Suzuki (2005), where the cumulative distribution for $\tau$ is obtained by integrating the escort probability and not the physical probability as here. These forms are related to the condition for the expectation value (Eq. 23) and has been the subject of detailed discussions (Wada and Scarfone, 2005; Ferri et al., 2005; Tsallis, 2009), where it was shown that the different forms are equivalent and can be transformed one into the other through simple operations defining $q \mathrm{~s}$ and $B_{q} \mathrm{~s}$. 
Finally, by applying the transformations for $q^{\prime}$ and $B_{q^{\prime}}$, the following form for $P(>\tau)$ is derived:

$P(>\tau)=\left[1-(1-q) B_{q} \tau\right]^{\frac{2-q}{1-q}}$.

We now apply this model to the scaled cumulative interevent time distribution of the earthquake activity at the West Corinth rift. We normalize interevent times $\tau$ (in seconds) as $\tau^{\prime}=\tau / \bar{\tau}$, where $\tau$ scales with the mean interevent time $\bar{\tau}=\left(t_{N}-t_{1}\right) /(N-1)$, leading to the scaled $P\left(>\tau^{\prime}\right)$. This is equivalent to the mean seismic rate $R=\bar{\tau}^{-1}$ that has been frequently used as a scaling factor for studying the interevent time distribution (e.g. Corral, 2004; Hainzl et al., 2006; Davidsen and Kwiatek, 2013). We also consider interevent times greater than $60 \mathrm{~s}$ due to possible incompleteness in lower time intervals, related to shadow effects in the seismograms. In Fig. 6a $P\left(>\tau^{\prime}\right)$ is plotted for the entire dataset and for $M \geq M_{\mathrm{c}}$. The cumulative distribution $P\left(>\tau^{\prime}\right)$ deviates at the intermediate and larger $\tau^{\prime}$ from the exponential function and rather exhibits a power-law long tail. The $q$-exponential distribution in the form of Eq. (30) provides on the other hand an excellent fit to the observed $P\left(>\tau^{\prime}\right)$ for the values of $q=1.25 \pm 0.02$ and $B_{q}=1.9 \pm 0.3$. This is also apparent from the $q$-logarithmic distribution $\ln _{q^{\prime}}\left(P\left(>\tau^{\prime}\right)\right)$ that is plotted in Fig. $6 \mathrm{~b}$, where the straight line is the $q$-exponential distribution. The proportion of the data that deviates from the $q$-exponential function at larger $\tau^{\prime}$ is less than $0.05 \%$ for $M \geq M_{\mathrm{c}}$.

We now turn our attention to the physical probability of the interevent times as it is interpreted in the probability density function. The probability density $p\left(\tau^{\prime}\right)$ for the normalized interevent times $\tau^{\prime}$ is obtained by dividing $\tau^{\prime}$ logarithmically and count the number of $\tau^{\prime}$ that falls into each bin. Then dividing this number by the total number of $\tau^{\prime}$ and the bin length, the normalized $p\left(\tau^{\prime}\right)$ is obtained over each bin.

Studying the normalized probability density, Corral (2004) analyzed the interevent time distribution for a number of seismic catalogues and showed that it can be described by a unique probability density function. This function can be well approximated by a gamma distribution of the form (Hainzl et al., 2006)

$p\left(\tau^{\prime}\right)=C \tau^{\prime(\gamma-1)} \exp \left(-\tau^{\prime} / \beta\right)$.

This result led Corral (2004) to claim that this empirical distribution is universal in the sense that it holds for regional and local scales and for a wide range of magnitudes when seismic activity is stationary, with constants $C=0.5 \pm 0.1, \gamma=$ $0.67 \pm 0.05$, and $\beta=1.58 \pm 0.15$. This distribution exhibits two regions, where short interevent times scale as a power law with exponent $1-\gamma$ and large interevent times decay exponentially. This defines a correlated behavior for earthquakes separated by short time intervals, while for larger time intervals earthquakes are independent.

In later studies the universal behavior in the interevent time distribution has been questioned (Davidsen and Goltz, 2004;

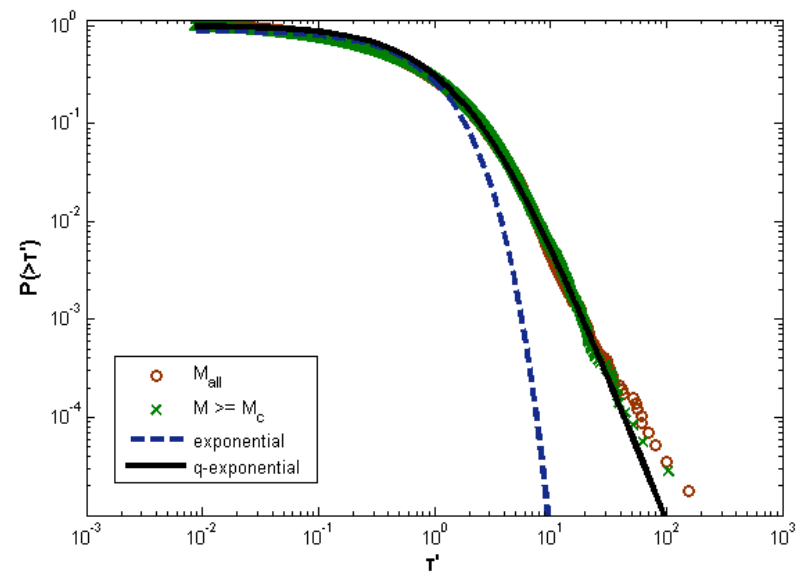

(a)

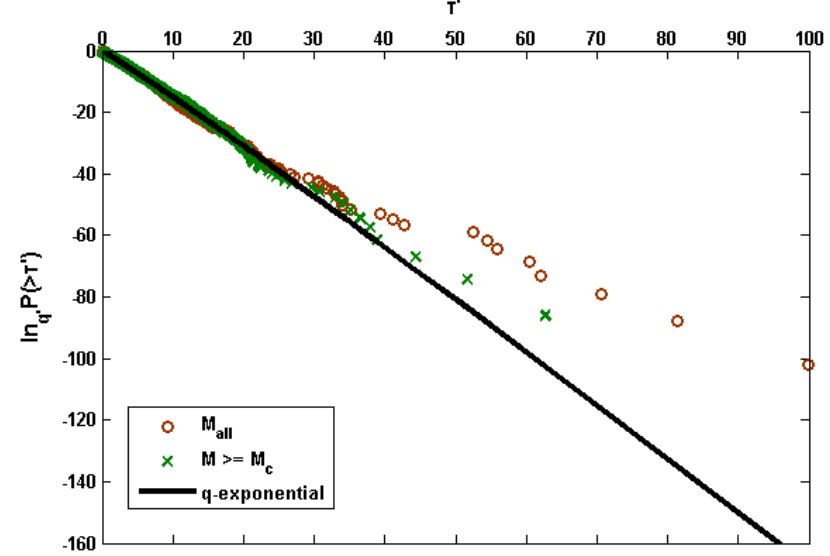

(b)

Fig. 6. (a) Log-log plot of $P\left(>\tau^{\prime}\right)$ for the entire dataset (circles) and for $M \geq M_{\mathrm{c}}$ (crosses). The solid line represents the $q$ exponential distribution of Eq. (30) for the values of $q=1.25 \pm 0.02$ and $B_{q}=1.9 \pm 0.3$. An exponential distribution (dashed line) is also plotted for comparison. (b) The corresponding $q$-logarithmic distributions $\ln _{q^{\prime}}\left(P\left(>\tau^{\prime}\right)\right)$ for the entire dataset (circles) and for $M \geq$ $M_{\mathrm{c}}$ (crosses), exhibiting correlation coefficients $\rho=-0.9842$ and $\rho=-0.9952$, respectively. The straight line is the $q$-exponential distribution.

Hainzl et al., 2006; Saichev and Sornette, 2006; Touati et al., 2009). In particular, deviations from this universal character seem to be controlled by the rate between correlated and uncorrelated activity at shorter and larger interevent times, respectively (Hainzl et al., 2006; Touati et al., 2009). Moreover, by analyzing simulations of the ETAS model, Touati et al. (2009) showed that the interevent time distribution can be best described by a mixture distribution between correlated events exhibiting gamma-distributed interevent times and uncorrelated events that are separated by larger time intervals decaying exponentially. Touati et al. (2009) also showed that the rate between correlated and uncorrelated events is strongly affected by the scale of the area that is considered. 
Larger areas have a high rate of independent events, while in smaller areas interevent times are highly nonrandom. More pronounced memory effects in local earthquake catalogues have been also reported by Livina et al. (2005).

Considering the high locality of the earthquake activity at the West Corinth rift, and taking into account the previous discussion, we can expect that the earthquake activity is highly nonrandom in this area. This is strongly supported by the results of DFA analysis and the $q$-exponential form of the cumulative interevent time distribution that indicated correlated behavior even at larger interevent times. This motivates us to propose a generalization of the gamma distribution (Eq. 31) as a possible scaling distribution of the probability density that does not decay exponentially but as a power-law. This distribution can be called $q$-generalized gamma distribution (Queirós, 2005) and has the form

$p\left(\tau^{\prime}\right)=C \tau^{\prime(\gamma-1)} \exp _{q}\left(-\tau^{\prime} / \theta\right)$.

Its connection to NESP is realized by the last $q$-exponential term, where the $q$-exponential function has been defined in Eq. (26). In the limit $q \rightarrow 1$, it recovers the ordinary gamma distribution. This is also known in statistics as the F-distribution (Marchand, 2003).

This distribution has been obtained theoretically by Queirós (2005), by considering a stochastic dynamical mechanism with memory effects that produces the observed variable. In our case, this mechanism can be considered the one that produces the successive earthquakes and thus the observed interevent time distribution. Then this process can be expressed in terms of a Feller process and its corresponding Fokker-Planck equation (see Queirós, 2005). For stationary periods, where the mean interevent time $\langle\tau\rangle$ does not fluctuate, the solution is a gamma distribution. On the other hand, if local fluctuations of mean $\langle\tau\rangle$ are produced, then the solution is the $q$-generalized gamma distribution of Eq. (32) (Queirós, 2005).

In Fig. 7 the normalized $p\left(\tau^{\prime}\right)$ is plotted for the entire dataset and for $M \geq M_{\mathrm{c}}$. The two distributions fall into the same curve and present two power-law regions at shorter and larger interevent times, respectively (Fig. 7a). Short interevent times decay as $\sim \tau^{\prime-0.65}$, larger ones as $\sim \tau^{\prime-3.45}$. In Fig. $7 \mathrm{~b}$ the three distributions, namely the $q$ exponential (Eq. 25), gamma (Eq. 31), and $q$-generalized gamma (Eq. 32), are fitted to the normalized $p\left(\tau^{\prime}\right)$. The $q$ exponential distribution, for the values of $Z_{q}=1.47, B=$ 1.34 , and $q=1.23$, provides a good fit at intermediate and larger $\tau^{\prime}$, while it deviates at short $\tau^{\prime}$. On the other hand, gamma distribution describes well the normalized $p\left(\tau^{\prime}\right)$ at short and intermediate interevent times, for the values of $C=0.35, \gamma=0.39, \beta=1.97$ and deviates at larger $\tau^{\prime}$. Then, the $q$-generalized gamma distribution provides an excellent fit for the entire range of $\tau^{\prime}$, for the values of $C=0.35, \gamma=$ $0.39, \theta=1.55$, and $q=1.23$. This result indicates the presence of correlated seismicity at all timescales and justifies

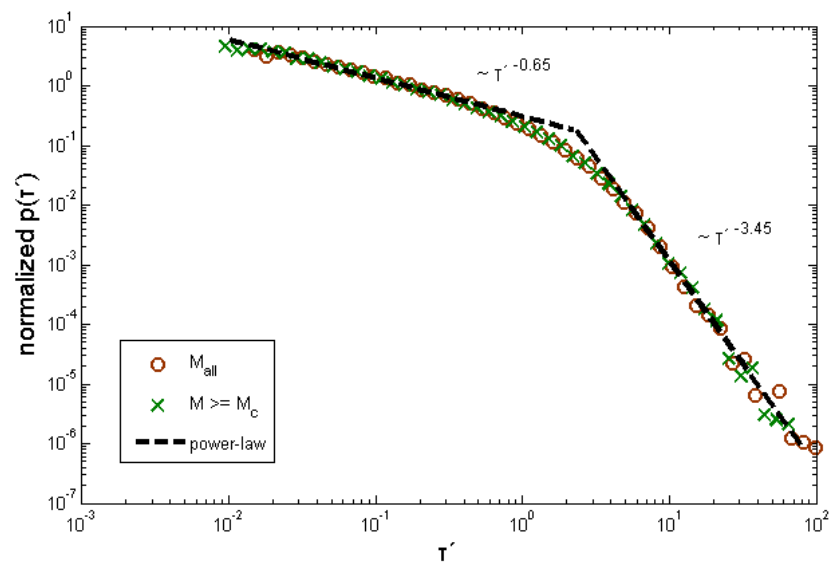

(a)

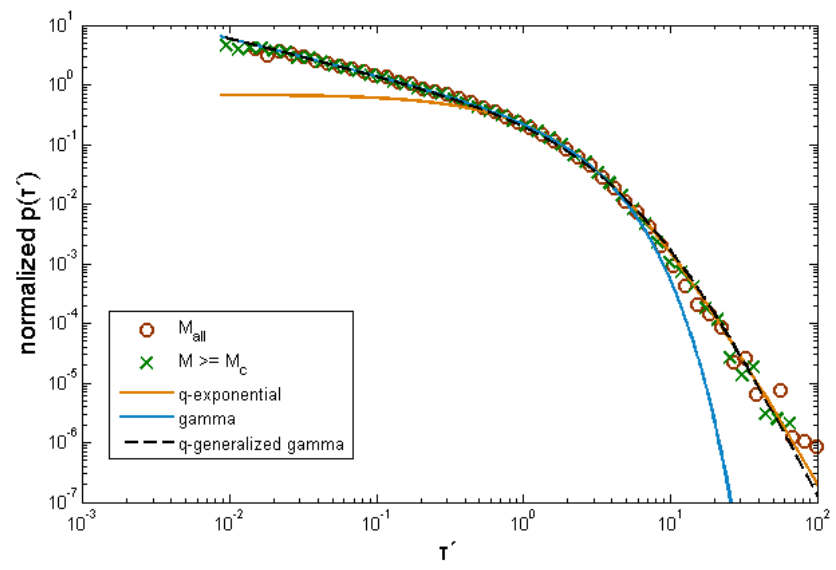

(b)

Fig. 7. Normalized probability density $p\left(\tau^{\prime}\right)$ for the scaled interevent times $\tau^{\prime}$. (a) Power-law fit. (b) The fitted curves that correspond to the $q$-exponential (Eq. 25) for the values of $Z_{q}=$ $1.47, B=1.34$, and $q=1.23$, gamma (Eq. 31) for the values of $C=0.35, \gamma=0.39, \beta=1.97$, and $q$-generalized gamma distribution (Eq. 32) for the values of $C=0.35, \gamma=0.39, \theta=1.55$, and $q=1.23$.

our approach. Moreover, the presence of scaling at short and large interevent times implies the multifractal character of the time evolution of the earthquake activity at the West Corinth rift that has been also reported in the work of Telesca et al. (2002).

\section{Conclusions}

In the present study the statistical properties of the earthquake activity at the West Corinth rift, a highly seismic zone in central Greece, have been investigated by means of non-extensive statistical physics (NESP) and detrended fluctuation analysis (DFA). The earthquake activity is highly localized in this area and exhibits long-range correlations between the times of the earthquakes events, as has been 
indicated by DFA. By applying a generalized physical model for earthquake dynamics, derived in a NESP formalism, the energy distribution function and the cumulative frequencymagnitude distribution were successfully described. Additionally, the properties of the cumulative distribution and the probability density of the interevent times were studied. The analysis showed that the cumulative distribution deviates from an ordinary exponential function that states uncorrelated behavior and can rather be well described by a $q$-exponential distribution that exhibits a power-law long tail. On the other hand, the probability density exhibits two power-law regions for the shorter and larger interevent times respectively, indicating that seismicity is correlated at alltime scales. The probability density, in comparison to a $q$ exponential and a gamma distribution, can be better described by a $q$-generalized gamma distribution that exhibits two power-law regions and accounts for nonstationarity and memory effects at all-time scales.

Summarizing, the present study indicates that the statistical properties of the earthquake activity at the West Corinth rift for the studied period are governed by long-range correlations and power-laws. These properties were successfully reproduced by means of NESP principles and should be taken into account in any probabilistic seismic hazard assessment for the area.

Acknowledgements. This work was supported by the THALES Program of the Ministry of Education of Greece and the European Union in the framework of the project entitled "Integrated understanding of Seismicity, using innovative Methodologies of Fracture mechanics along with Earthquake and non-extensive statistical physics - Application to the geodynamic system of the Hellenic Arc. SEISMO FEAR HELLARC”.

Edited by: G. Zöller

Reviewed by: three anonymous referees

\section{References}

Abe, S. and Suzuki, N.: Law for the distance between successive earthquakes, J. Geophys. Res., 108, 2113, doi:10.1029/2002JB002220, 2003.

Abe, S. and Suzuki, N.: Scale-free statistics of time interval between successive earthquakes, Physica A, 350, 588-596, doi:10.1016/j.physa.2004.10.040, 2005.

Ambraseys, N. N. and Jackson, J. A.: Seismicity and associated strain of central Greece between 1890 and 1988, Geophys. J. Int., 101, 663-708, doi:10.1111/j.1365-246X.1990.tb05577.x, 1990.

Armijo, R., Meyer, B., King, G. C. P., Rigo, A., and Papanastassiou, D.: Quaternary evolution of the Corinth Rift and its implications for the Late Cenozoic evolution of the Aegean, Geophys. J. Int., 126, 11-53, doi:10.1111/j.1365-246X.1996.tb05264.x, 1996.

Bak, P. and Tang, C.: Earthquakes as a self-organized critical phenomenon, J. Geophys. Res., 94, 15635-15637, doi:10.1029/JB094iB11p15635, 1989.
Bak, P., Christensen, K., Danon, L., and Scanlon, T.: Unified scaling law for earthquakes, Phys. Rev. Lett., 88, 178501, doi:10.1103/PhysRevLett.88.178501, 2002.

Bashan, A., Bartsch, R., Kantelhardt, J. W., and Havlin, S.: Comparison of detrending methods for fluctuation analysis, Physica A, 387, 5080-5090, doi:10.1016/j.jhydrol.2011.01.032, 2008.

Bernard, P., Briole, P., Meyer, B., Lyon-Caen, H., Gomez, J.-M., Tiberi, C., Berge, C., Cattin, R., Hatzfeld, D., Lachet, C., Lebrun, B., Deschamps, A., Courboulex, F., Larroque, C., Rigo, A., Massonnet, D., Papadimitriou, P., Kassaras, J., Diagourtas, D., Makropoulos, K., Veis, G., Papazisi, E., Mitsakaki, C., Karakostas, V., Papadimitriou, E., Papanastassiou, D., Chouliaras, M., and Stavrakakis, G.: A low angle normal fault earthquake: the $\mathrm{M}_{S}=6.2$, June 1995 Aigion earthquake (Greece), J. Seismol., 1, 131-150, doi:10.1023/A:1009795618839, 1997.

Bourouis, S. and Cornet, F. H.: Microseismic activity and fluid fault interactions: Some results from the Corinth Rift Laboratory (CRL), Greece, Geophys. J. Int., 178, 561-580, doi:10.1111/j.1365-246X.2009.04148.x, 2009.

Burridge, R. and Knopoff, L.: Model and theoretical seismicity, B. Seismol. Soc. Am., 57, 341-371, 1967.

Chen, C. C., Telesca, L .C., Lee, T., and Sun, Y. S.: Statistical physics of landslides: New paradigm, Europhys. Lett., 95, 49001, doi:10.1209/0295-5075/95/49001, 2011.

Chen, Z., Ivanov, P. C., Hu, K., and Stanley, H. E.: Effect of nonstationarities on detrended fluctuation analysis, Phys. Rev. E, 65, 041107/1-041107/15, doi:10.1103/PhysRevE.65.041107, 2002.

Clarke, P. J., Davies, R. R., England, P. C., Parsons, B. E., Billiris, H., Paradissis, D., Veis, G., Denys, P. H., Cross, P. A., Ashkenazi, V., and Bingley, R.: Geodetic estimate of seismic hazard in the Gulf of Korinthos, Geophys. Res. Lett., 24, 1303-1306, doi:10.1029/97GL01042, 1997.

Corral, A.: Long-term clustering, scaling, and universality in the temporal occurrence of earthquakes, Phys. Rev. Lett., 92, 108501, doi:10.1103/PhysRevLett.92.108501, 2004.

Darooneh, A. H. and Dadashinia, C.: Analysis of the spatial and temporal distributions between successive earthquakes: Nonextensive statistical mechanics viewpoint, Physica A, 387, 36473654, doi:10.1016/j.physa.2008.02.050, 2008.

Davidsen, J. and Goltz, C.: Are seismic waiting time distributions universal?, Geophys. Res. Lett., 31, L21612, doi:10.1029/2004GL020892, 2004.

Davidsen, J. and Kwiatek, G.: Earthquake interevent time distribution for induced micro-, nano-, and picoseismicity, Phys. Rev. Lett., 11, 068501, doi:10.1103/PhysRevLett.110.068501, 2013.

De Rubeis, V., Hallgas, R., Loreto, V., Paladin, G., Pietronero, L., and Tosi, P.: Self-affine asperity model for earthquakes, Phys. Rev. Lett., 76, 2599-2602, doi:10.1103/PhysRevLett.76.2599, 1996.

Ferri, G. L., Martínez, S., and Plastino, A.: Equivalence of the four versions of Tsallis's statistics, J. Stat. Mech.-Theory E, 2005, P04009, doi:10.1088/1742-5468/2005/04/P04009, 2005.

Gell-Mann, M. and Tsallis, C. (Eds.): Nonextensive Entropy - Interdisciplinary Applications, Oxford University Press, New York, 2004.

Gutenberg, B. and Richter, C. F.: Frequency of earthquakes in California, B. Seismol. Soc. Am., 34-4, 185-188, 1944. 
Hainzl, S., Scherbaum, F., and Beauval, C.: Estimating background activity based on interevent-time distribution, B. Seismol. Soc. Am., 96, 313-320, doi:10.1785/0120050053, 2006.

Hu, K., Ivanov, P. C., Chen, Z., Carpena, P., and Stanley, H. E.: Effect of trends on detrended fluctuation analysis, Phys. Rev. E, 64, 011114/1-011114/19, doi:10.1103/PhysRevE.64.011114, 2001.

Kanamori, H.: Quantification of earthquakes, Nature, 271, 411-414, doi:10.1038/271411a0, 1978

Kantelhardt, J. W., Koscielny-Bunde, E., Rego, H. H. A., Havlin, S., and Bunde, A.: Detecting long-range correlations with detrended fluctuation analysis, Physica A, 295, 441-454, doi:10.1016/S0378-4371(01)00144-3, 2001.

Keilis-Borok, V. I.: The lithosphere of the earth as a nonlinear system with implications for earthquake prediction, Rev. Geophys., 28, 19-34, doi:10.1029/RG028i001p00019, 1990.

Krajcinovic, D. and Van Mier, J. G. M.: Damage and Fracture of Disordered Materials, Springer-Verlag, New York, 2000.

Lay, T. and Wallace, T. C.: Modern Global Seismology, Academic Press, New York, 1995.

Lennartz, S., Livina, V. N., Bunde, A., and Havlin, S.: Longterm memory in earthquakes and the distribution of interoccurrence times, Europhys. Lett., 81, 69001, doi:10.1209/02955075/81/69001, 2008.

Lennartz, S., Bunde, A., and Turcotte, D. L.: Modelling seismic catalogues by cascade models: Do we need long-term magnitude correlations?, Geophys. J. Int., 184, 1214-1222, doi:10.1111/j.1365-246X.2010.04902.x, 2011.

Livina, V. N., Havlin, S., and Bunde, A.: Memory in the occurrence of earthquakes, Phys. Rev. Lett., 95, 208501, doi:10.1103/PhysRevLett.95.208501, 2005.

Main, I.: Statistical physics, seismogenesis, and seismic hazard, Rev. Geophys., 34, 433-462, doi:10.1029/96RG02808, 1996.

Makropoulos, K. C., Drakopoulos, J. K., and Latousakis, J. B.: A revised and extended earthquake catalogue for Greece since 1900, Geophys. J. Int., 98, 391-394, doi:10.1111/j.1365246X.1989.tb03360.x, 1989.

Mandelbrot, B. B.: The Fractal Geometry of Nature, Freeman, San Francisco, 1983.

Marchand J.-P.: Distributions, North-Holland, Amsterdam, 2003.

Pacchiani, F. and Lyon-Caen, H.: Geometry and spatio-temporal evolution of the 2001 Agios Ioanis earthquake swarm (Corinth rift, Greece), Geophys. J. Int., 180, 59-72, doi:10.1111/j.1365246X.2009.04409.x, 2010.

Peng, C.-K., Buldyrev, S. V., Havlin, S., Simons, M., Stanley, H. E., and Goldberger, A. L.: Mosaic organization of DNA nucleotides, Phys. Rev. E, 49, 1685-1689, doi:10.1103/PhysRevE.49.1685, 1994.

Peng, C.-K., Havlin, S., Stanley, H. E., and Goldberger, A. L.: Quantification of scaling exponents and crossover phenomena in nonstationary heartbeat time series, Chaos, 5, 82-87, doi:10.1063/1.166141, 1995.

Picoli, S., Mendes, R. S., Malacarne, L. C., and Santos, R. P. B.: qdistributions in complex systems: A brief review, Braz. J. Phys., 39, 468-474, 2009.

Queirós, S. M. D.: On the emergence of a generalised gamma distribution. Application to traded volume in financial markets, Europhys. Lett., 71, 339-345, doi:10.1209/epl/i2005-10109-0, 2005.

Rundle, J. B., Turcotte, D. L., Shcherbakov, R., Klein, W., and Sammis, C.: Statistical physics approach to understanding the multi- scale dynamics of earthquake fault systems, Rev. Geophys., 41, 1019, doi:10.1029/2003RG000135, 2003.

Saichev, A. and Sornette, D.: "Universal" distribution of interearthquake times explained, Phys. Rev. Lett., 97, 078501, doi:10.1103/PhysRevLett.97.078501, 2006.

Silva, R., Franca, G. S., Vilar, C. S., and Alcaniz, J. S.: Nonextensive models for earthquakes, Phys. Rev. E, 73, 026102, doi:10.1103/PhysRevE.73.026102, 2006.

Sornette, D.: Critical phenomena in natural sciences, chaos, fractals, self-organization and disorder: concepts and tools, Springer, Heidelberg, 2nd Edn., 528 pp., 2004.

Sotolongo-Costa, O. and Posadas, A.: Fragment-asperity interaction model for earthquakes, Phys. Rev. Lett., 92, 048501 , doi:10.1103/PhysRevLett.92.048501, 2004.

Telesca, L.: Nonextensive analysis of seismic sequences, Physica A, 389, 1911-1914, doi:10.1016/j.physa.2010.01.012, 2010a.

Telesca, L.: Analysis of Italian seismicity by using a nonextensive approach, Tectonophysics, 494, 155-162, doi:10.1016/j.tecto.2010.09.012, 2010b.

Telesca, L.: Tsallis-based nonextensive analysis of the Southern California seismicity, Entropy, 13, 1267-1280, doi:10.3390/e13071267, 2011.

Telesca, L.: Maximum likelihood estimation of the nonextensive parameters of the earthquake cumulative magnitude distribution, B. Seismol. Soc. Am., 102, 886-891, doi:10.1785/?0120110093, 2012.

Telesca, L. and Lovallo, M.: Non-uniform scaling features in central Italy seismicity: A non-linear approach in investigating seismic patterns and detection of possible earthquake precursors, Geophys. Res. Lett., 36, L01308, doi:10.1029/2008GL036247, 2009.

Telesca, L., Cuomo, V., Lapenna, V., Vallianatos, F., and Drakatos, G.: Analysis of the temporal properties of Greek aftershock sequences, Tectonophysics, 341, 163-178, doi:10.1016/S00401951(01)00221-9, 2001.

Telesca, L., Lapenna, V., and Vallianatos, F.: Monofractal and multifractal approaches in investigating scaling properties in temporal patterns of the 1983-2000 seismicity in the western Corinth graben, Greece, Phys. Earth Planet. In., 131, 63-79, doi:10.1016/S0031-9201(02)00014-6, 2002.

Telesca, L., Lapenna, V., and Macchiato, M.: Spatial variability of the time-correlated behaviour in Italian seismicity, Earth Planet. Sc. Lett., 212, 279-290, doi:10.1016/S0012-821X(03)00286-3, 2003.

Touati, S., Naylor, M., and Main, I. G.: Origin and nonuniversality of the earthquake interevent time distribution, Phys. Rev. Lett., 102, 168501, doi:10.1103/PhysRevLett.102.168501, 2009.

Tsallis, C.: Possible generalization of Boltzmann-Gibbs statistics, J. Stat. Phys., 52, 479-487, doi:10.1007/BF01016429, 1988.

Tsallis, C.: Introduction to nonextensive statistical mechanics: Approaching a complex world, Springer, Berlin, 378 pp., 2009.

Turcotte, D. L.: Fractals and Chaos in Geology and Geophysics, Cambridge University Press, Cambridge, UK, 2nd Edn., 398 pp., 1997.

Vallianatos, F.: A non-extensive approach to risk assessment, Nat. Hazards Earth Syst. Sci., 9, 211-216, doi:10.5194/nhess-9-2112009, 2009.

Vallianatos, F.: A non-extensive statistical physics approach to the polarity reversals of the geomagnetic field, Physica A, 390, 1773-1778, doi:10.1016/j.physa.2010.12.040, 2011. 
Vallianatos, F.: On the statistical physics of rockfalls: A nonextensive view, Europhys. Lett., 101, 10007, doi:10.1209/02955075/101/10007, 2013.

Vallianatos, F. and Sammonds, P.: Is plate tectonics a case of non-extensive thermodynamics?, Physica A, 389, 4989-4993, doi:10.1016/j.physa.2010.06.056, 2010.

Vallianatos, F. and Sammonds, P.: A non-extensive statistics of the fault-population at the Valles Marineris extensional province, Mars, Tectonophysics, 509, 50-54, doi:10.1016/j.tecto.2011.06.001, 2011.

Vallianatos, F. and Sammonds, P.: Evidence of non-extensive statistical physics of the lithospheric instability approaching the 2004 Sumatran-Andaman and 2011 Honshu mega-earthquakes, Tectonophysics, 590, 52-58, doi:10.1016/j.tecto.2013.01.009, 2013.

Vallianatos, F. and Triantis, D.: Is Pressure Stimulated Current Relaxation in Amphibolite a Case of Non-Extensivity?, Europhys. Lett., 99, 18006, doi:10.1209/0295-5075/99/18006, 2012.

Vallianatos, F., Triantis, D., and Sammonds, P.: Non-extensivity of the isothermal depolarization relaxation currents in uniaxial compressed rocks, Europhys. Lett., 94, 68008, doi:10.1209/0295-5075/94/68008, 2011.

Vallianatos, F., Benson, P., Meredith, P., and Sammonds, P.: Experimental evidence of a non-extensive statistical physics behavior of fracture in triaxially deformed Etna basalt using acoustic emissions, Europhys. Lett., 97, 58002, doi:10.1209/02955075/97/58002, 2012a.
Vallianatos, F., Michas, G., Papadakis, G., and Sammonds, P.: A non-extensive statistical physics view to the spatiotemporal properties of the June 1995, Aigion earthquake (M6.2) aftershock sequence (West Corinth rift, Greece), Acta Geophys., 60, 758-768, doi:10.2478/s11600-012-0011-2, 2012b.

Vallianatos, F., Michas, G., Papadakis, G., and Tzanis, A.: Evidence of non-extensivity in the seismicity observed during the 20112012 unrest at the Santorini volcanic complex, Greece, Nat. Hazards Earth Syst. Sci., 13, 177-185, doi:10.5194/nhess-13-1772013, 2013.

Varotsos, P. A., Sarlis, N. V., and Skordas, E. S.: Long-range correlations in the electric signals that precede rupture, Phys. Rev. E, 66, 011902/1-011902/7, doi:10.1103/PhysRevE.78.059901, 2002.

Varotsos, P. A., Sarlis, N. V., Tanaka, H. K., and Skordas, E. S.: Similarity of fluctuations in correlated systems: The case of seismicity, Phys. Rev. E, 72, 041103, doi:10.1103/PhysRevE.72.041103, 2005.

Vilar, C. S., França, G. S., Silva, R., and Alcaniz, J. S.: Nonextensivity in geological faults?, Physica A, 377, 285-290, doi:10.1016/j.physa.2006.11.017, 2007.

Wada, T. and Scarfone, A. M.: Connection between Tsallis' formalisms employing the standard linear average energy and ones employing the normalized q-average energy, Phys. Lett. A, 335, 351-362, doi:10.1016/j.physleta.2004.12.054, 2005.

Xu, L., Ivanov, P. C., Hu, K., Chen, Z., Carbone, A., and Stanley, H. E.: Quantifying signals with power-law correlations: A comparative study of detrended fluctuation analysis and detrended moving average techniques, Phys. Rev. E, 71, 051101, doi:10.1103/PhysRevE.71.051101, 2005. 